\title{
GENERALIZED BRONCHIECTASIS ASSOCIATED WITH DEFICIENCY OF CARTILAGE IN THE BRONCHIAL TREE
}

\author{
BY
}

HOWARD WILLIAMS and PETER CAMPBELL

From the Departments of Clinical Research and Pathology, Royal Children's Hospital, Melbourne

(RECEIVED FOR PUBLICATION JULY 20, 1959)

Bronchiectasis may occasionally affect almost the entire bronchial tree. Such changes can be the result either of developmental defects of the bronchial tree or of acquired inflammatory disease. For example, cystic lung and cystic bronchiectasis can result from both causes.

The purpose of the present paper is to record five patients with a most unusual type of generalized bronchiectasis, and to submit evidence that its basis is deficiency of the cartilage of the bronchial walls, probably developmental in origin.

\section{Material and Methods}

The five patients have been studied clinically for periods between three and nine years. Radiological, bronchoscopic, bronchographic and bacteriological examinations have been carried out at suitable intervals. The lungs of one of the two patients who died were examined in the fresh state and then fixed by injecting the bronchi with formalin. Studies consisted of macroscopic dissection of several segmental bronchi and their divisions, and serial histological sections of four lung segments (apical, anterior and posterior basic and lingula of the left lung). The stains used were haematoxylin and eosin, Verhoeff van Gieson and periodic acid stain of Schiff. The bronchi were identified numerically by counting distally from the segmental bronchus (Hayward and Reid, 1952); see Appendix.

\section{Clinical Features}

Case 1. J.G. In October, 1949, a boy aged $2 \frac{3}{4}$ years was referred to the Royal Children's Hospital with a cough, irregular fever and wheezy breathing, which developed following an attack of morbilli six weeks previously. Before this he was well and had no serious illnesses. His doctor had made a diagnosis of unresolved pneumonia and asthma, and had unsuccessfully treated him with antibiotics and antispasmodics. He was found to be thin, with slightly cyanosed lips, and his breathing was difficult and audibly wheezy. His chest was barrelshaped, and inspiratory and expiratory rhonchi and fine crepitations were heard over the entire chest. A radiograph of the chest showed prominent hilar shadows and bronchovascular markings, some haziness in the right middle lobe and lingula and slight pulmonary emphysema. His Mantoux test 1:1000 OT was negative and Streptococcus viridans was grown from a small blob of expectorated mucopus. Bronchoscopic examination was normal apart from some muco-purulent secretion in both bronchi. A remarkable bronchographic picture was seen on the x-ray screen, the whole bronchial tree from the first division of the segmental bronchus to the fifth or eighth division (depending on the size of the segment) expanded and narrowed with inspiration and expiration (Fig. 1a and b), while the fourth to sixth or eighth divisions of the right bronchial tree were like a series of elongated balloons which were being inflated and deflated (Fig. 2a and b). These changes were more pronounced in the right than in the left lung. At the site of bronchial branching the movement was not so marked. Apart from one or two small areas, no oil entered the fine divisions of the bronchial tree, even though dye was still present in the bronchial tree $\mathbf{4 0}$ hours later (Fig. 3a and $b$ ).

Treatment with penicillin and sulphonamides and postural coughing led to slight improvement in his general condition but the cough and wheeze persisted and he periodically had attacks of fever and malaise. As he grew older his symptoms lessened and his general health improved. Nine years after the onset he is in fair health but is unable to run without becoming very shortwinded, has a constant slight inspiratory and expiratory wheeze and cough. His chest is barrel-shaped with a pigeon deformity and Harrison's sulcus. 


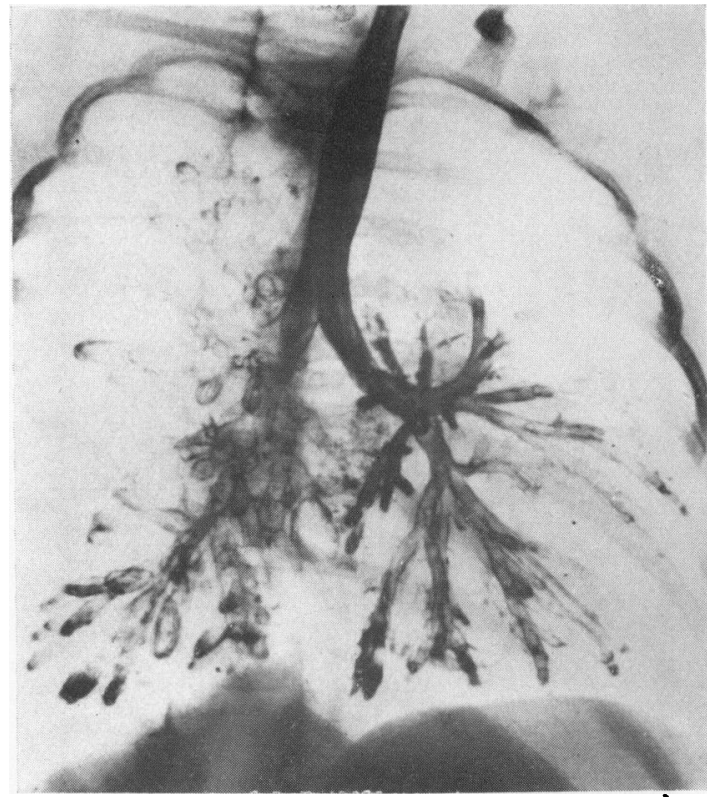

Fig. 1a.-Case 1. J.G. Bronchogram in inspiration showing 'cystic' dilatation of right bronchial tree and cylindrical dilatation of left.

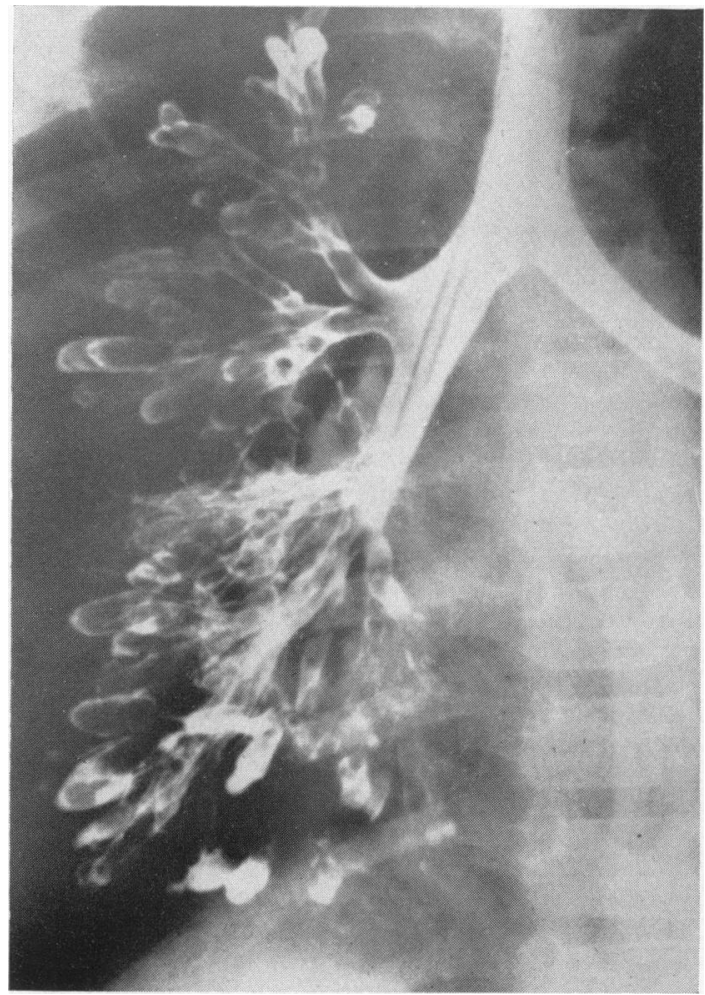

Fig. 2a.-Case 1. J.G. Bronchogram of right bronchial tree in inspiration showing gross bronchial dilatation beyond segmental bronchi but no bronchiolar filling.

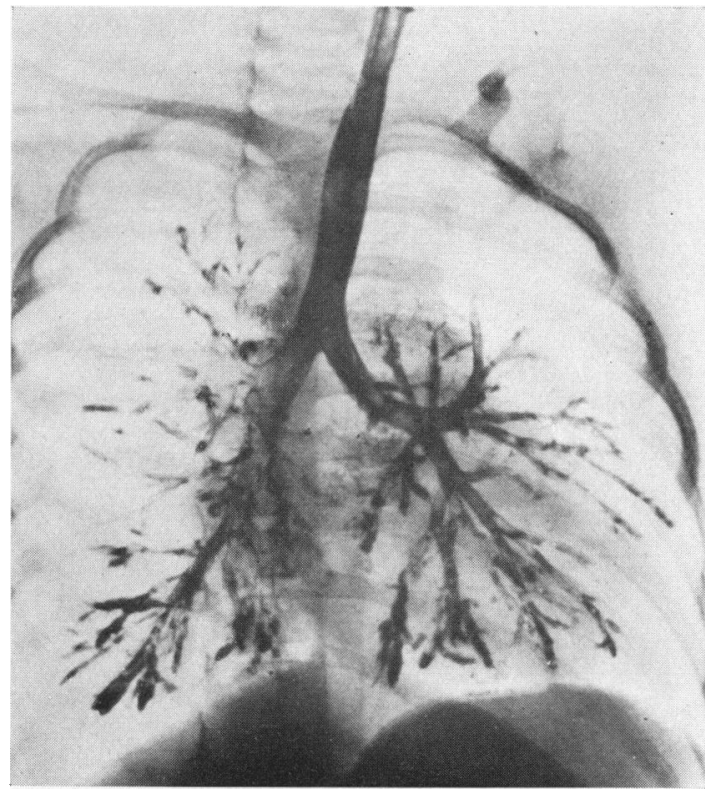

Fig. 1b.-Case 1. J.G. Bronchogram in expiration, showing collapse of bronchi.

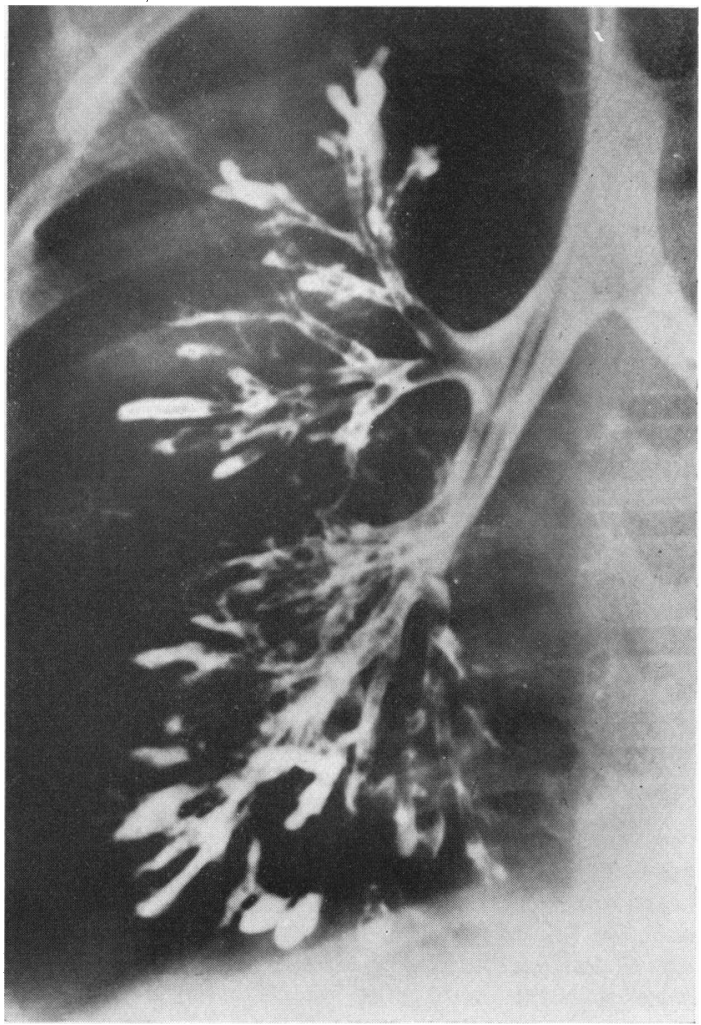

FIG. 2b.-Case 1. J.G. Bronchogram of right bronchial tree in expiration showing collapse of bronchi. 


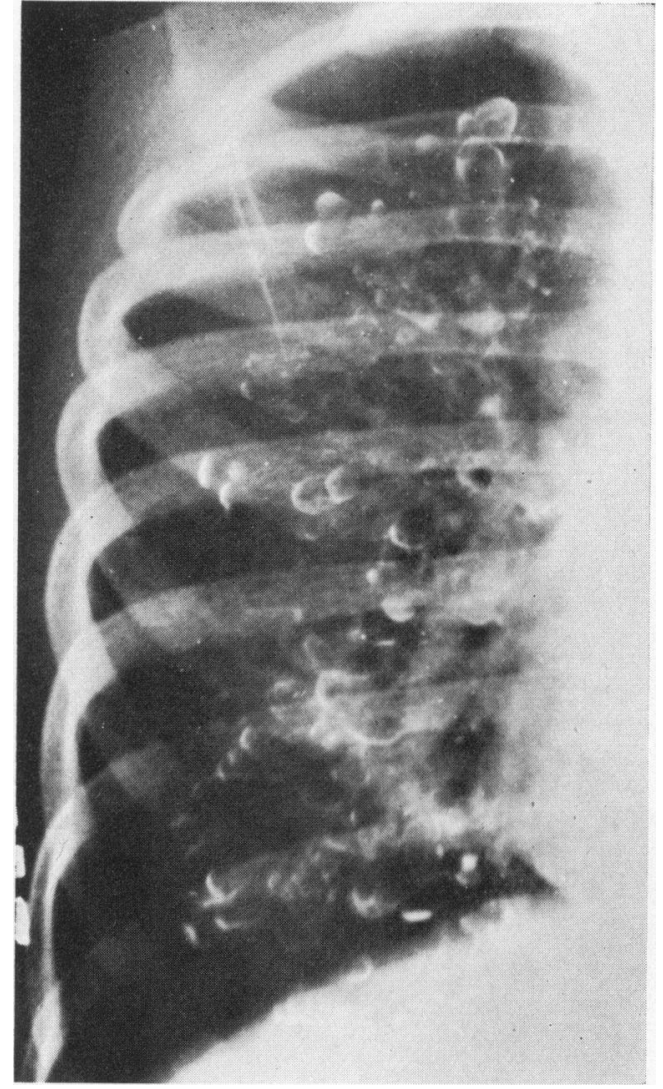

FIG. 3a.-Case 1. J.G. Film in inspiration taken 40 hours after Fig. 2a showing retention of dye and no filling of small bronchi.

Case 2. D.S., the first baby of healthy parents, made normal progress until the age of 10 months when he developed a running nose, cough and wheezy breathing. A diagnosis of asthmatic bronchitis was made, but treatment with antibiotics and antispasmodics failed to relieve his symptoms. The cough, wheezing and febrile attacks gradually became worse and his general health deteriorated.

At the age of 2 years 8 months when he was admitted to the Royal Children's Hospital in July 1952 he was ill with distressed wheezy breathing and slight cyanosis of lips and ears. He had a barrel-shaped chest with bilateral Harrison's sulcus, the air entry over the entire chest was poor, and inspiratory and expiratory rhonchi and numerous fine crepitations were heard over the whole lungs. The heart was displaced to the left and the percussion note over the left upper lobe was impaired. The abdomen was distended, the fingers clubbed and he expectorated some mucopus, culture of which grew Staphylococcus aureus. A chest radiograph showed collapse of the left upper lobe and mediastinal shift to the left, and the peripheral lung field of the right lung and the left lower lobe

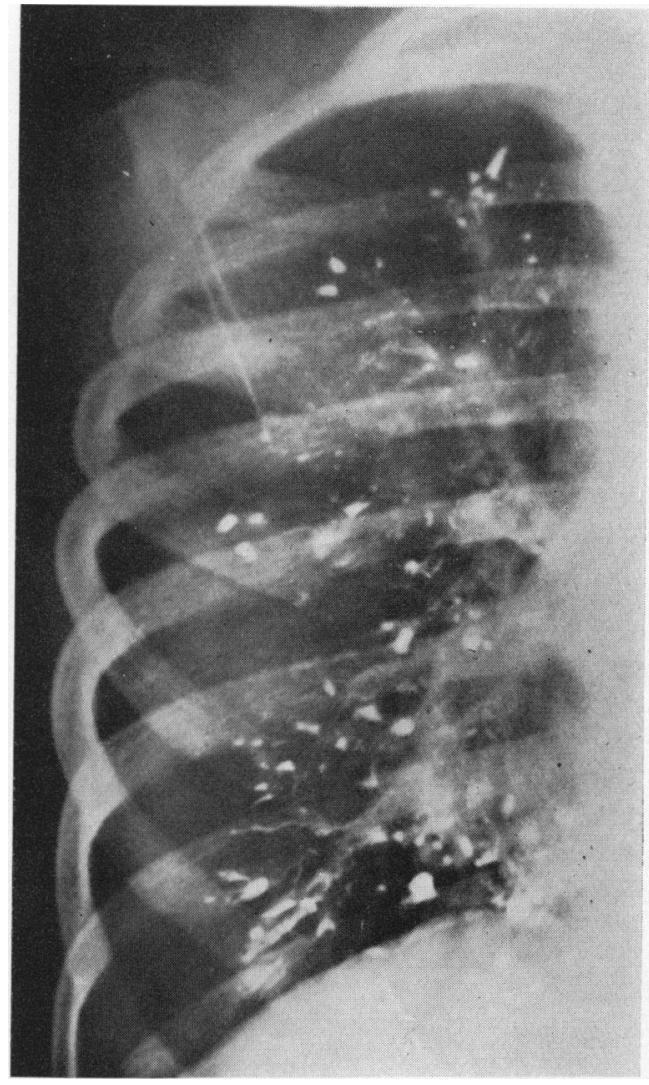

FIG. 3b.-Case 1. J.G. Film in expiration taken 40 hours after Fig. 2a showing collapse of bronchi.

appeared emphysematous. Bronchoscopy was normal apart from a small amount of mucopus in both bronchi. Bronchographic examination was almost identical with that in Case 1, but the bronchiectatic changes were most pronounced on the left side where the left lung was collapsed. Expansion and partial collapse were observed from the first division of the segmental bronchus to the fifth or eighth division, according to the segment, but no dye entered the bronchiolar tree.

Treatment with antibiotics, antispasmodics and postural coughing afforded only slight relief. His condition gradually deteriorated and he died of respiratory failure at the age of 3 years 2 months. Autopsy was refused.

Case 3. J.C. was a healthy baby of healthy parents until she developed pink disease at the age of 14 months. She was left with a slight cough and occasionally had a slight wheeze. At the age of 2 years 3 months she developed measles following which her cough became worse, her breathing difficult and wheezy, her stools pale and offensive and her body wasted. A diagnosis of 
fibrocystic disease of the pancreas was made. Following treatment with chemotherapy she very slowly improved.

When seen at the Royal Children's Hospital in May 1953 at the age of 4 years she appeared in fair general health, had a rather barrel-shaped chest, a slight wheeze and produced a little mucopus on posture. Impaired percussion note and tubular breathing were heard over the left lower lobe and scattered crepitations over the entire chest. A radiograph of the chest showed increase in the broncho-vascular markings, collapse of the left lower lobe and somewhat emphysematous peripheral lung fields. Duodenal enzymes and fat balance were normal. Bronchoscopy was normal apart from a little mucopus in the left lower lobe bronchus. The bronchogram showed changes similar to those in Cases 1 and 2 with the exception that in the right lower lobe and pectoral segment of the left upper lobe the bronchial calibre changes during respiration were not so pronounced and there was filling of some of the smaller bronchi and the bronchiolar tree.

From the age of 4 years up to $9 \frac{1}{2}$ years she slowly and steadily improved in general health. She has a very slight cough at present and usually no sputum and only wheezes and coughs sputum if she develops a cold or has a feverish attack. These attacks used to occur every few months but now she has them only occasionally. She can run but gets short of wind easily. A ventilatory capacity test at the age of 9 years showed figures indicative of a moderately severe obstructive type of ventilatory defect.

Case 4. A.D., a healthy baby of healthy parents, made normal progress until the age of 10 months when she contracted morbilli. After this she developed a cough, rapid wheezy breathing and would not eat. As these symptoms persisted she was referred to the Royal Children's Hospital in November 1955 aged 1 year with a diagnosis of unresolved broncho-pneumonia. She was moderately well nourished, had slightly rapid laboured respirations with an audible inspiratory and expiratory wheeze. Numerous inspiratory and expiratory rhonchi and fine crepitations were heard all over the chest. A radiograph showed prominent bronchovascular markings and some emphysema in the peripheral lung fields. Culture of a cough swab yielded Staph. aureus and Proteus. Treatment with one of the tetracycline group of drugs resulted in some general improvement but the cough and wheeze persisted, she continued to run episodes of fever and her signs remained unchanged. Bronchoscopy at the age of 2 years was normal apart from some mucopus in both bronchi. A bronchogram showed symmetrical expansion and collapse of the bronchial tree from the region of the first segmental divisions down to the fifth or eighth branching, according to the segment. Apart from a few areas the dye did not enter small bronchi or bronchioles.

During the two years follow-up her general health has slowly improved. She still has a slight cough and an audible inspiratory and expiratory wheeze, but during the last year has only had two mild feverish attacks. Her chest is deformed with a marked pigeon chest, bilateral
Harrison's sulcus and she breathes with the upper part. She becomes short-winded if she attempts to run.

Case 5. A.W., a premature baby weighing $4 \mathrm{lb} .7 \mathrm{oz}$, developed a cough and wheeze at the age of $2 \frac{1}{2}$ months. The cough and wheeze persisted, he ran frequent febrile episodes and was said to be cyanosed at times. When first seen at the Royal Children's Hospital in December 1952 at the age of $2 \frac{1}{2}$ years he was a thin, poorly nourished child (weight $20 \mathrm{lb}$.) with slightly cyanosed lips and ears. His breathing was difficult and there was a moderately loud inspiratory and expiratory wheeze. The chest was barrel-shaped, movement being predominantly in the upper portion, and numerous fine crepitations and rhonchi were heard all over the chest. No other abnormalities were found. A radiograph of the chest showed increased bronchovascular markings and very few vascular markings in the peripheral lung fields. His Mantoux test 1: 1000 OT was negative, his stools did not show any fat globules and culture of a cough swab yielded Staph. aureus. Bronchoscopic examination was normal apart from some reddening of the bronchial mucosa and a small amount of purulent exudate in both bronchi. The bronchogram showed that the segmental bronchi down to the fifth or eighth divisions, according to the segment, dilated and narrowed with inspiration and expiration (Fig. $4 \mathrm{a}$ and b), but not to the same degree as in the first three patients. Several areas in both lungs showed some bronchiolar filling. Dye was still present in the dilated bronchi 48 hours after instillation.

During the next two years until his death at the age of 4 years 8 months he had a constant cough, increasingly difficult breathing with cyanosis and frequent episodes of fever. Gradually he developed pulmonary hypertension, his heart enlarged, and an angiogram showed some dilatation of the right auricle, right ventricle and pulmonary artery, while the pulmonary tree showed irregular tortuous small arterioles with delayed passage of dye. Cardiac catheterization showed moderate pulmonary hypertension.

\section{Morbid Anatomical Features of Case 5}

Pathological changes were confined to the respiratory and cardiovascular systems. In the fresh state the lungs appeared congested and felt nodular. Bronchi distal to the main segmental divisions were dilated and the mucosa of all bronchi was oedematous and inflamed. The pulmonary arteries were dilated and slightly atheromatous, and the heart showed moderate hypertrophy and dilatation of the right ventricle. After formalin fixation further macroscopic and microscopic studies were carried out.

Macroscopic Appearances. The segmental bronchi were normal, but the second and third divisions were dilated, being of similar calibre to the segmental bronchus. Many of the dilated lateral divisions suddenly tapered to a fine thread barely possessing 


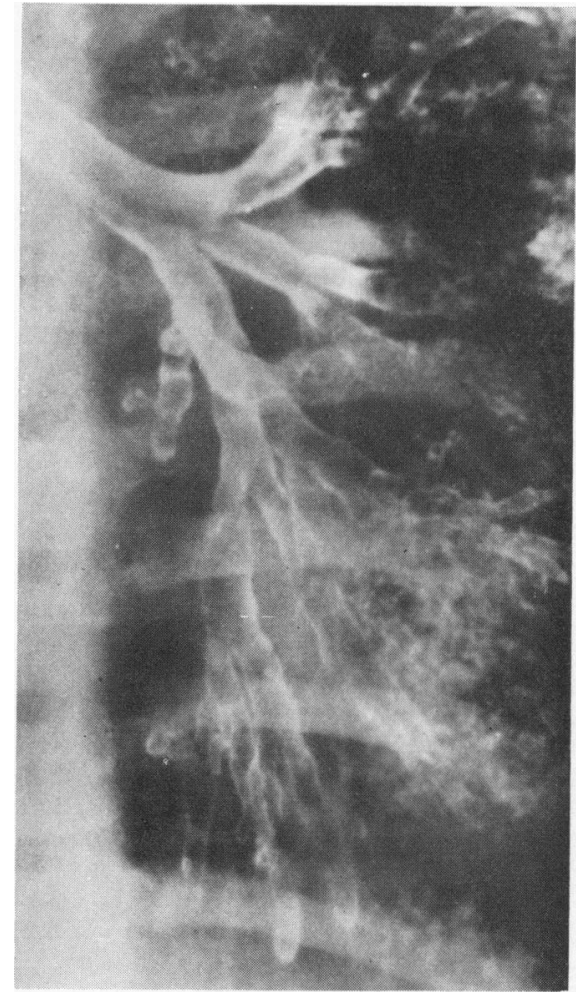

Frg. 4a.-Case 5. A.W. Bronchogram showing bronchial dilatation in left lower lobe. Film in inspiration.

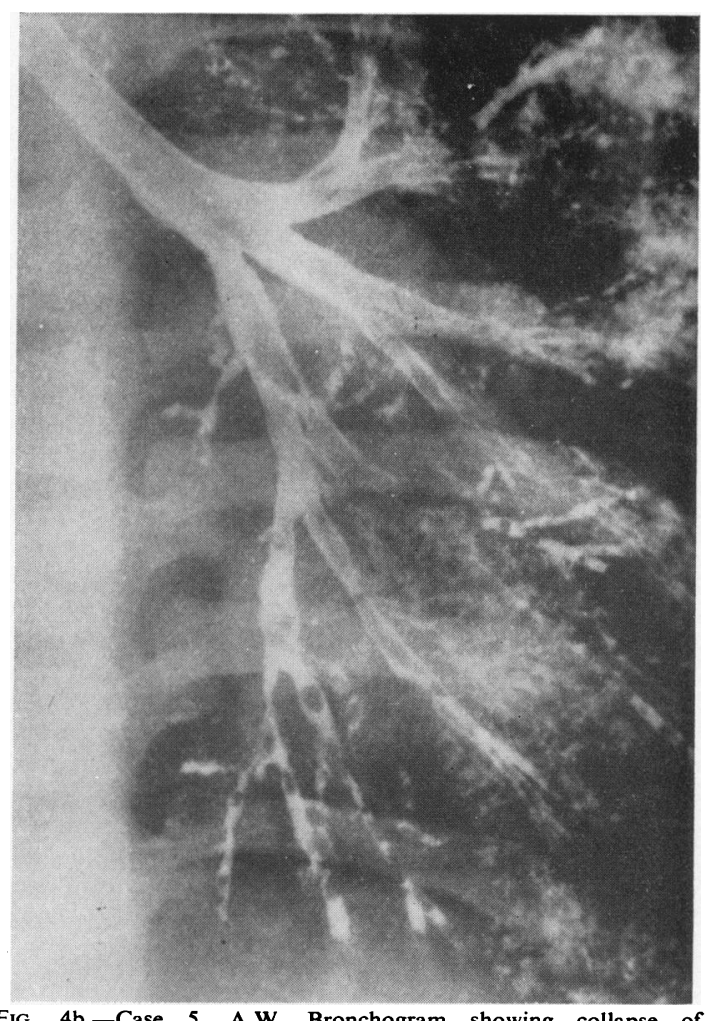

FIG. 4b.-Case 5. A.W. Bronchogram showing collapse of bronchi in left lower lobe. Film in expiration.

a lumen. Divisions of the axial bronchi distal to the third division gradually tapered, but were still greatly dilated compared with the normal controls (Fig. 5a and $b$ ). The most striking feature was the extreme softness of the bronchial walls, which, despite formalin fixation, were as flabby as blood vessels. In nearly all of the bronchi dissected the transition from firm rigid walls to soft easily compressible structures was sudden and occurred along the course of the second or third division. From this point to the periphery the walls remained soft, except where division occurred when a slight resistance could be felt in the acute angle of the bifurcation. In several dissections it was

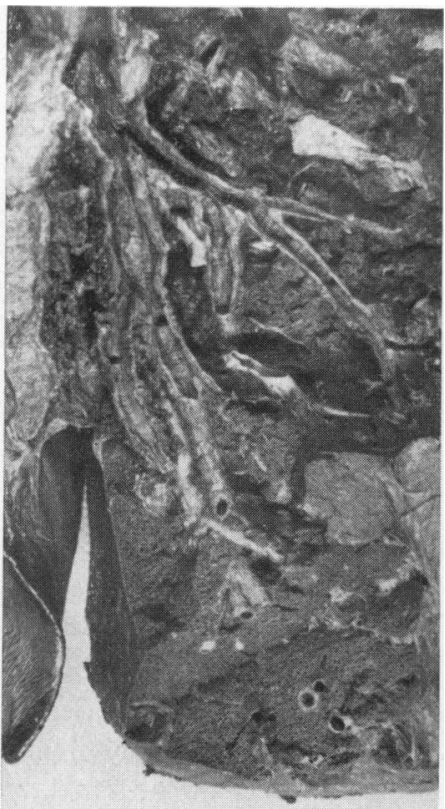

Fig. 5a.-Case 5. A.W. Dissection showing dilated bronchi, partly unroofed, of lingula and pectoral segments. (Slightly

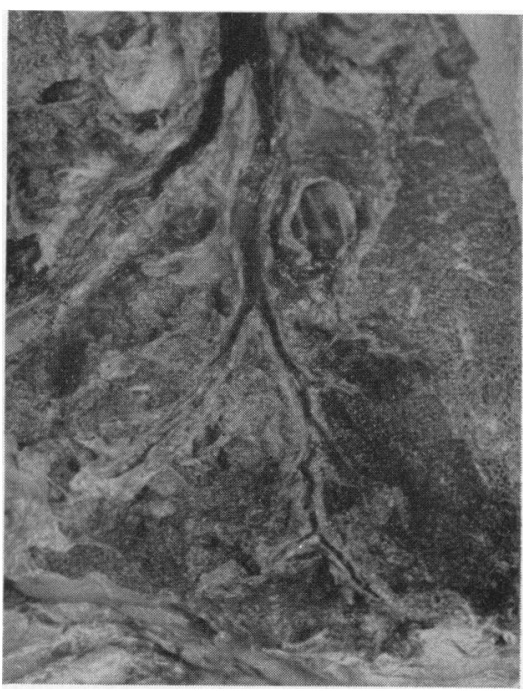

FIG. 5b.-Dissection of normal anterior basic bronchus and its divisions. (Slightly reduced.) 
difficult to trace an axial bronchus to the periphery, bronchial branching being dichotomous and forming two divisions of identical calibre. In many instances, however, a true axial bronchus was present (see Appendix).

Microscopical Findings. These were as follows. Segmental Bronchi (First Division Bronchi). The mucosa showed pseudostratified ciliated columnar epithelium with areas of squamous metaplasia (mucosa less folded than normal) resting on a narrow condensed band of collagen 7-20 $\mu$ in thickness. Foci of lymphocytes were present with little apparent relation to the nature or state of the mucosal cells (viz., pseudostratified, ciliated or nonciliated, squamous metaplasia).

Muscle coat was present in all bronchi examined. It was very thin in places $(30-40 \mu)$ but elsewhere was quite thick $(200-300 \mu)$. It was often thin next to cartilage plaques and thicker where cartilage was absent. Beneath the muscle and merging with it was a network of collagen and elastic fibres, thicker and more prominent in areas deficient in cartilage.

Individual plaques of cartilage varied in size and appeared 'chunky', i.e. they were not long and thin as in the normal; in cross-section their size varied from $700 \mu$ to $2,500 \mu$ and, as in the normal, their edges merged with the surrounding fibro-elastic network.

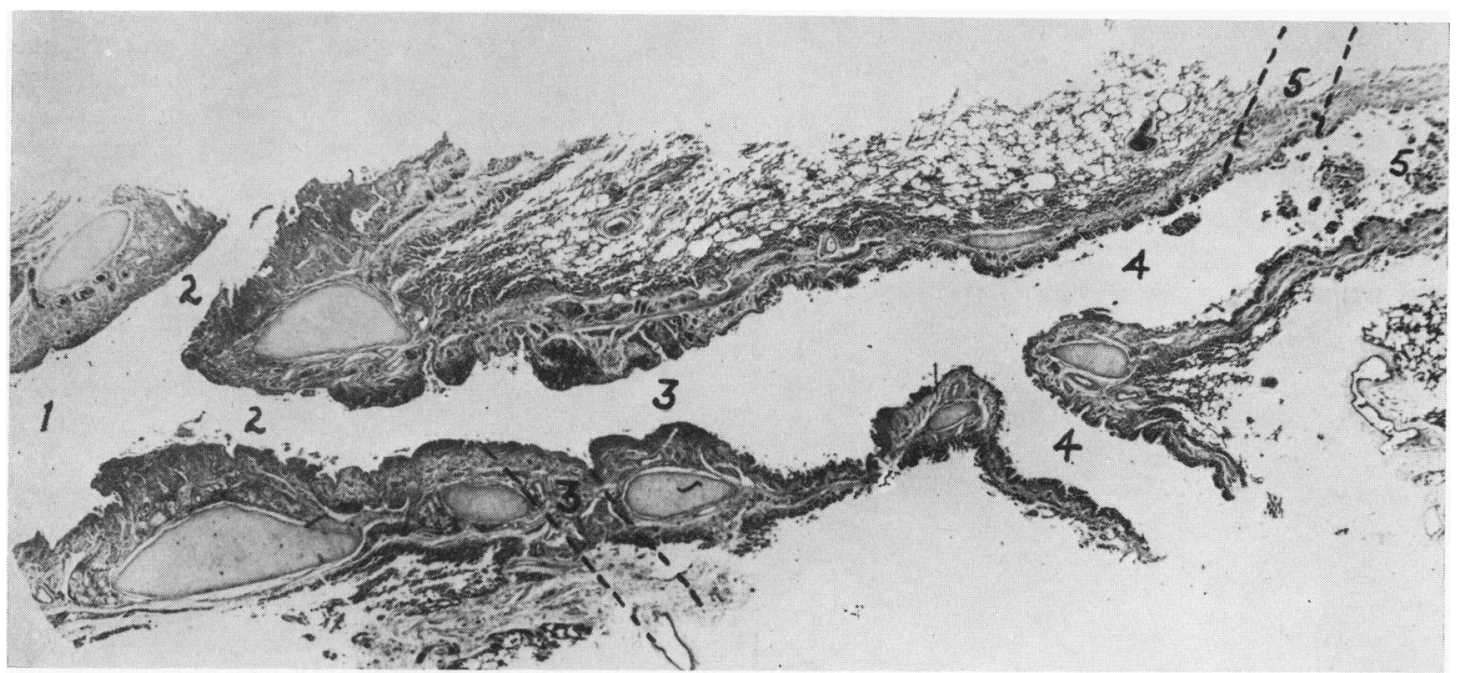

FIG. 6a.-Case 5. A.W. Longitudinal section of segmental bronchus showing cartilage distribution. (Note two bronchi arose out of plane of section. Bronchial divisions are numbered.)

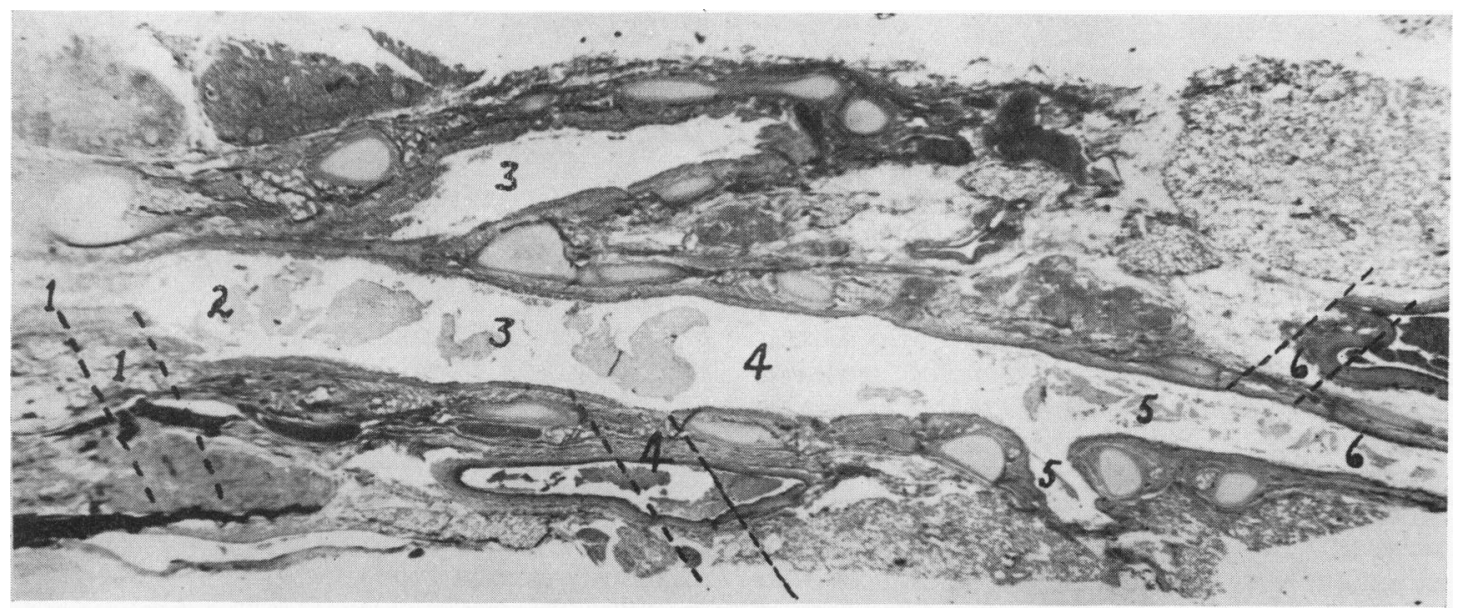

Fig. 6b.-Longitudinal section of segmental bronchus showing cartilage distribution in the normal. Bronchial divisions are numbered. Three bronchi arose out of plane of section. 


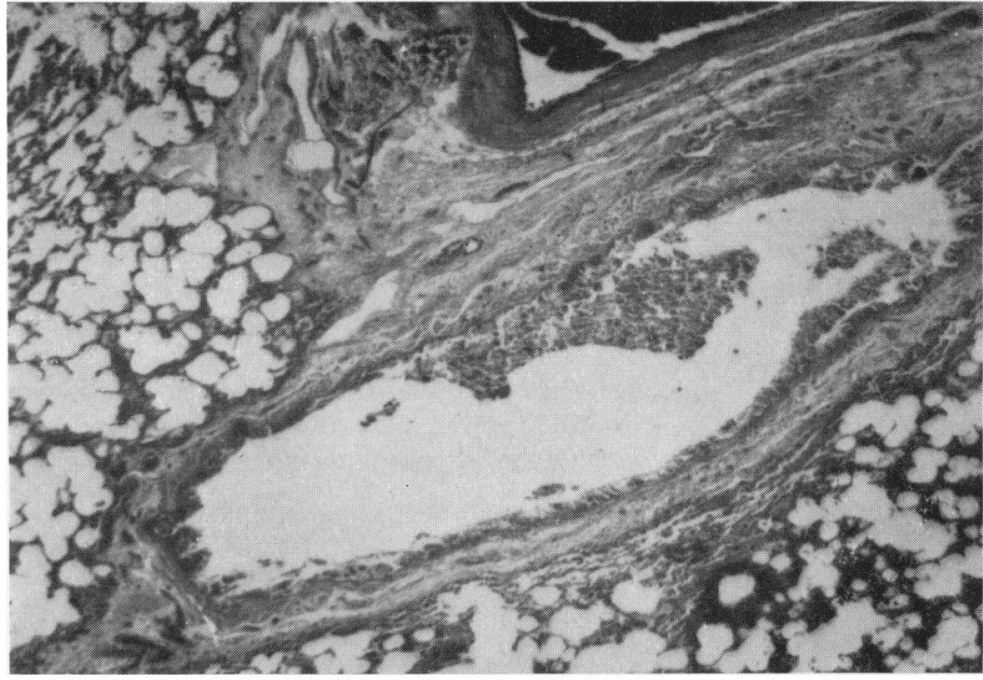

FIG. 7a.-Case 5. A.W. Showing a cross-section of a fifth division axial bronchus. Note complete absence of cartilage in wall. $(\times 25$.)

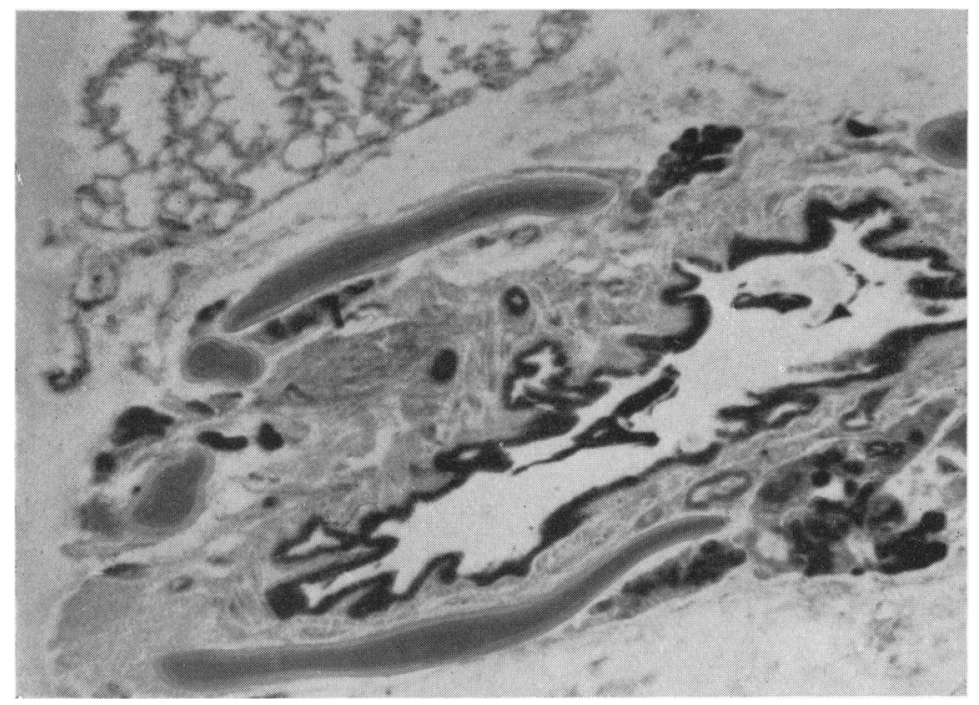

Fig. 7b.-Normal control, showing cross-section of fifth division axial bronchus. Note cartilage present. $(\times 25$.

Sub-segmental Bronchi (Second and Distal Divisions). More numerous areas of squamous metaplasia were present in the mucosa, otherwise the pattern was similar to that in the segmental bronchus.

Muscle was invariably present, sometimes thin $(30-40 \mu)$ but quite often normal in appearance and thickness. Isolated areas of small round cell infiltration were present, some of which were associated with partial fibrous replacement of muscle.
One must distinguish between the axial bronchus and its lateral divisions, all lateral divisions being devoid of cartilage whereas in occasional cases the third and even fourth division axial bronchi contained isolated cartilage plates. Thus, examination of sections from the anterior basic bronchus (left lung) showed cartilage distribution as follows. The segmental bronchus and the first, second and third division bronchi contained cartilage. In the third division bronchi, the plaques were widely separated and were small. In the fourth division bronchus, very occasional plaques only were visible. Examination of the lingula bronchus showed cartilage as far down as the third division but it was absent distal to this. All other bronchi from the end of the second division showed walls devoid of cartilage except at each point of bifurcation where a small nub of cartilage was present in all cases down as far as the fifth, sixth and seventh division bronchi (Figs. 6a and b; 7a and b).

The following changes were found in the more peripheral parts of the lungs. Bronchiolar obliteration was often severe and extensive (Fig. 8). Bronchioles were far less numerous than in the normal controls. Some that had been recently obliterated were still recognizable, but many were represented only by small areas of scarring. Emphysema was focal, related to obliterated bronchioles and moderately severe. Vascular
thickening was slight, involving the medium-sized and small arterioles.

\section{Discussion}

The clinical features in all five children were very similar. The disease commenced in infancy, either insidiously as mild bronchitis or more acutely with morbilli. Persistent cough, wheezy difficult breathing, recurrent febrile episodes and widespread 


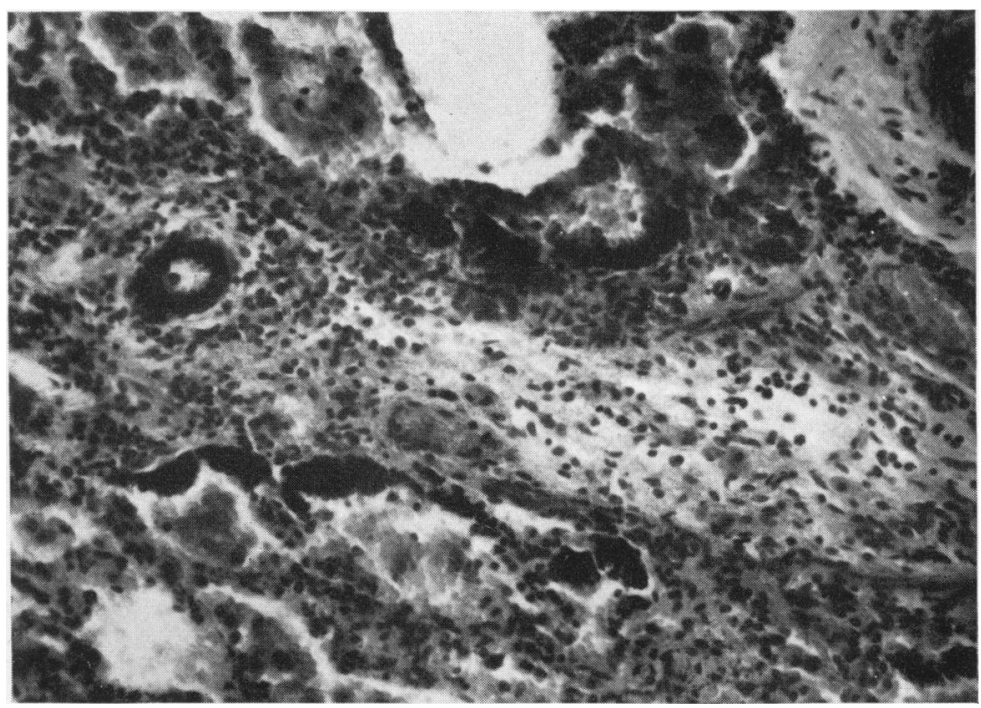

FIg. 8.-Case 5. A.W. Showing bronchiolar obliteration. $(\times 125$.

inspiratory and expiratory rhonchi and crepitations were the main clinical features. The chest was deformed as a result of diffuse bronchial and bronchiolar obstruction and pulmonary emphysema. As the clinical pattern and physical signs were those of chronic or subacute bronchiolitis with recurrent exacerbations, it was no wonder that a diagnosis of asthmatic bronchitis, or non-specific bronchiolitis, or fibrocystic disease of the pancreas was made.

The course of the disease varied considerably. In two patients it was steadily downhill, death occurring at the ages of $4 \frac{3}{4}$ and 3 years. Both patients died of progressive respiratory failure, the result of chronic infective bronchiolitis and pulmonary hypertension. One of the three living children is in good health, the only symptoms being a little cough and breathlessness out of proportion to the degree of physical activity. The other two are severely limited, any active exertion resulting in breathlessness. As these children have grown older, infection has become less and their general health correspondingly improved.

The clinical features and bronchographic appearances of Case 1 suggested that expansion and narrowing of the bronchial walls with respiration was due to considerable structural weakness. Lack of cartilage seemed to be the probable structural weakness as similar expansion and collapse of the walls of the trachea and main bronchi had been observed when cartilage was absent from maldevelopment (Ferguson and Neuhauser, 1944; Evans, 1949; and Holinger and Johnston, 1957).
The gross morbid anatomical findings in Case 5 revealed dilatation of the sub-segmental bronchial divisions which were soft to feel and easily compressed, a marked contrast to the normal controls. Serial histological examination disclosed that the cartilage was deficient or absent along the walls in most cases from the third division down, but in small numbers was present in the angle of bifurcation of the bronchi. This deficiency in cartilage in the bronchial walls readily explains the physiological defect observed under the $x$-ray screen.

The problem is whether this defect is a developmental one, or whether it is secondary to infection. The available evidence favours a primary developmental origin for the following reasons. The lesions were surprisingly uniform, involving almost the entire bronchial tree from the second or third segmental division downwards. The principal defect in the structure of the bronchial wall was in the amount and distribution of cartilage. Had the cartilage been destroyed by inflammation, other structures would almost certainly have been affected, e.g. muscle and elastic tissue, but there was little evidence of this. Again, if inflammation had caused dissolution of cartilage in the walls of the bronchi, one would expect to find several bronchi in which no cartilage at all was present. In fact, however, in all cases where a division occurred one could find a small piece of cartilage at the bifurcation, although the walls themselves remained deficient. Examination of the bronchograms shows a 'nipping in' at the site of branching of the bronchi, 
corresponding presumably with this small piece of cartilage.

In acquired bronchiectatic lesions cartilage destruction rarely extends up to the first and second segmental divisions, and then never in a uniform manner. Several patients examined in the present group soon after the onset of the disease showed gross bronchographic changes. The duration and the severity of the clinical infection was too short and too mild to result in such gross structural changes in the bronchial walls or to destroy the cartilage and cause 'flapping' during respiration. In no single case of bronchiectasis due to acquired disease have we seen changes comparable with those in these patients. We therefore presume that the cartilaginous defect is a developmental one and that the symptoms of chronic cough, wheezing febrile episodes and later pulmonary emphysema, pulmonary hypertension and respiration failure in two patients were consequent on chronic secondary infection in the bronchiolar tree.

\section{Summary}

Five children with an unusual type of generalized bronchiectasis are described.

The clinical features of chronic cough, wheezy breathing and febrile attacks associated with widespread clinical signs in the chest and pulmonary emphysema were easily confused with asthmatic bronchitis, or chronic non-specific bronchiolitis or fibrocystic disease of the pancreas.

The clinical and radiological features in the five patients and the morbid anatomical findings in one suggest that the underlying basis is maldevelopment in the cartilage of the bronchial tree.

\section{REFERENCES}

Evans, W. A. (1949). Congenital obstructions of the respiratory tract. I. Tracheal malformations. Amer. J. Roentgenol., $62,167$.

Ferguson. C. F. and Neuhauser, E. D. B. (1944). Congenital absence of the lung (agenesis) and other anomalies of the tracheobronchial tree. Amer. J. Roentgenol., 52, 459.

Holinger, P. H. and Johnston, K. C. (1957). Clinical aspects of congenital anomalies of the trachea and bronchi. Dis. Chest, 31, 613 .

\section{APPENDIX}

The lungs of five children of approximately the same age as Case 5 were examined by macroscopic dissection and by serial section of segmental bronchi and their divisions to establish a normal pattern for children of this age group (Gray (1954), Hayek (1953), Miller (1937)). The results are summarized below.

\section{Macroscopic Findings}

Method. Fixed lungs were dissected with fine scissors commencing at the segmental bronchus, which was called the first division bronchus (see Fig. 9). Any branches arising from this were counted and the dissection was carried as far as possible into the periphery of the lung.

Findings. The number of divisions of any segmental bronchus depended on the distance between the origin of the bronchus and the lung tissue it supplied. It was greater in longer bronchi than in short, e.g. lingula, with 10 macroscopically visible bronchial divisions, and pectoral, with five to six macroscopically visible bronchial divisions.

Divisions were of two types. Larger bronchi arose at a very acute angle. Small bronchi generally arose at a much greater angle from the parent trunk (lateral bronchi of Hayward and Reid, 1952).

The concept of an axial bronchus (Hayward and Reid, 1952) was not always easy to demonstrate. True dichotomous branching was common, the resulting bronchi being of similar calibre and coursing to the periphery, each giving off approximately the same number of divisions (see Fig. 5b).

In general, however, a main or 'axial' bronchus could be followed from which lesser bronchi arose.

The bronchi in the fixed state were firm and elastic.

Circumferential cartilage plaques were present in all segmental bronchi and distally for three to six divisions, depending on the length of the axial bronchus.

Isolated cartilage plaques occurred in the walls of bronchi from the fourth to the tenth divisions, although they were difficult to see in the more peripheral divisions and their presence had to be confirmed by histological examination.

\section{Microscopic Findings}

Serial sections were examined in continuity and all the branches arising from an axial bronchus were counted and an accurate assessment made of their cartilage content. The findings confirmed those made macroscopically.

Mucosa. Regular ciliated columnar epithelium pseudostratified down as far as the smaller bronchioles when it tended to become simple columnar in type. Mucosa rested on a condensed layer of collagen and merged with the underlying collagen network around mucous glands, muscle and cartilage.

Muscle. This occurred in regular fasciculi beneath the mucosa and varied in thickness with the bronchi exam- 


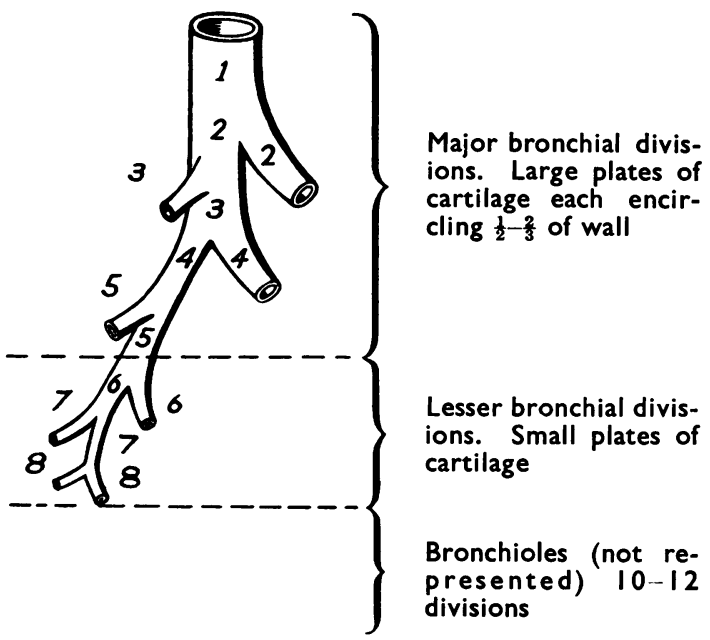

L. Post Basic Br.

Fig. 9.-Sketch of a typical normal bronchus and its divisions. Diagram shows method of numbering bronchi and distribution of cartilage in them.

ined (from $50 \mu$ up to $500 \mu$ ). Muscle composed only a small portion of the thickness of the walls of the larger bronchi $\left(\frac{1}{8}\right.$ to $\left.\frac{1}{10}\right)$ and slightly more $\left(\frac{1}{5}\right.$ to $\left.\frac{1}{4}\right)$ of the more distal divisions.

Cartilage. In the larger bronchi (in general divisions 1-6) this was present as large plaques each partly encircling the lumen (so-called circumferential cartilage plaques). Their thickness varied greatly, being greater the larger the bronchus, and generally ranging from 300-1,000 $\mu$ (Fig. 6b).

In the lesser bronchi (axial divisions distal to the fourth or fifth, and most of the lateral bronchi) the cartilage was distributed sporadically in the wall as isolated bars joined by a condensation of collagen and elastic fibres. A cross-section of such a bronchus would reveal two to four small plaques around the circumference. Their thickness averaged $100-300 \mu$. Such cartilage plaques could be found distally as far as the sixth to tenth division, depending on which segment was being examined.

Bronchioles. The transition from bronchus to bronchiole was usually clear. Two criteria were used $(a)$ cessation of cartilage, and $(b)$ relation to surrounding lung. Thus, bronchi were always surrounded by a condensate of collagen in which ran several vessels, lymphatics and nerves, whereas bronchioles lay in intimate relation with lung parenchyma and lacked this collagenous mantle (Engel, 1947). In general the small peripheral bronchi contained cartilage in their walls, but this was felt to be a less reliable criterion than the latter.

On these criteria bronchiolar divisions numbered $10-12$ in most cases.

\section{REFERENCES}

Engel, S. (1947). The Child's Lung. Arnold, London. Ch. 2. The bronchial and bronchiolar distribution and arrangement. Ch. 3. The developmental histology of the bronchial tree. Ch. 8. Bronchiectasis.

Gray, H. (1954). Gray's Anatomy. 31st ed. Ed. by T. B. Johnston and J. Whillis. Longmans, Green and Co., London. Pp. 1293-1299. The trachea and bronchi.

Hayek, H. Von (1953). Die menschliche Lunge. Springer, Berlin. In text.

Hayward, J. and Reid, L. M. (1952). The cartilage of the intrapulmonary bronchi in normal lungs, in bronchiectasis and in massive collapse. Thorax, 7,98.

Miller, W. S. (1937). The Lung. Thomas, Springfield, Ill. Ch. 3. Intrapulmonary bronchi and bronchioli. 